\title{
Price of Anarchy for Cognitive MAC Games
}

\author{
Lok Man Law ${ }^{1}$, Jianwei Huang ${ }^{1}$, Mingyan Liu ${ }^{2}$, Shuo-yen $\mathrm{Li}^{1}$ \\ ${ }^{1}$ Information Engineering Department, Chinese University of Hong Kong, $\{1$ lm007, jwhuang, bobli $\} @$ ie.cuhk.edu.hk \\ ${ }^{2}$ Department of Electrical Engineering and Computer Science, University of Michigan, mingyan@eecs.umich.edu
}

\begin{abstract}
In this paper, we model and analyze the interactions between secondary users in a spectrum overlay cognitive system as a cognitive MAC game. In this game, each secondary user can sense (and transmit) one of several channels, the availability of each channel is determined by the activity of the corresponding primary user. We show that this game belongs to the class of congestion game and thus there exists at least one Nash Equilibrium. We focus on analyzing the worst case efficiency loss (i.e., price of anarchy) at any Nash Equilibrium of such a game. Closed-form expressions of price of anarchy are derived for both symmetric and asymmetric games, with arbitrary channel and user heterogeneity. Several insights are also derived in terms of how to design a better cognitive radio system with less severe efficiency loss.
\end{abstract}

\section{BACKGROUND AND CONTRIBUTIONS}

The wireless spectrum resource is scarce due to rapid development of wireless technologies and exploding increase of wireless applications. Recently, cognitive radio has emerged as a promising technology to alleviate this problem. In cognitive radio networks, the existing licensees (i.e., primary users or PUs) of the spectrum share the spectrum with the opportunistic users (i.e., secondary users or SUs) to improve the spectrum efficiency (e.g., [13], [14]). There are different interaction modes between the PUs and SUs. In the spectrum underlay mode, both PUs and SUs transmit simultaneously, and the PUs closely monitor and control the interferences generated by the SUs to be below certain threshold (e.g., [1]-[3]). In the spectrum overlay case, the SUs sense the spectrum, locate the spectrum holes, and only transmit in the time, location, and frequency band where the PUs are not active (e.g., [4]-[6]). This paper studies the medium access control (MAC) game among several SUs in a spectrum overlay scenario.

Most existing results on spectrum overlay focused the study on the spectrum access of either a single SU or several SUs who do not closely interact with each other (e.g., [18], [19]). Instead we analyze the close interactions among several SUs who want to compete for the access in a heterogeneous multichannel environment. Each SU decides which channel to sense (and transmit) to maximize his expected data rate, taking into consideration the channel rate, the PU's activity, and other SUs' possible choices. We show that such interactions can be modeled as a congestion game, which has been studied mostly in the contexts of wireline resource allocations (e.g., [7]-[9]). Using congestion game to model wireless resource allocations only starts to receive attention recently (e.g., [12]).

The key question we want to answer is the following: how bad can the selfish behaviors of the SUs be in the cognitive
MAC game? More specifically, if we look at the ratio between the total rate achieved under any Nash Equilibrium of the game and the maximum total rate achievable under a coordinated MAC protocol, how small can it be? The worst case ratio is normally called Price of Anarchy (PoA) in the networking literature [11], [17]. There exists a large volume of literature in terms of studying PoA in the contexts of wireline networking, e.g., [15], [16], [18]. In particular, the bounds of PoA for congestion games have been previously computed in [10], [17]. In [10], the PoA is computed for finite games where all users have the same payoff for the same chosen strategy. Here we allow a more general model where users have different payoffs for the same chosen strategy. In [17], PoA is calculated for a wireline network in terms of total latency incurred by the flows, while we compute PoA in terms of total expected transmission rate in a wireless network.

The main results and contributions of this paper include:

- General network model: we consider both symmetric and asymmetric cognitive MAC games. In the more general asymmetric case, we allow each SU to have independent evaluation of the channels' rates. This allows a complete heterogeneous network model which is realistic but difficult to analyze.

- Exact characterization of PoA: we derive exact closedform solutions of the PoA, instead of giving (possibly loose) upper or lower bounds.

- Novel analysis techniques: we characterize the PoA without explicitly solving for the maximum possible total rate for each case. This technique turns out to be quite powerful in the general and difficult cases.

- Insights for a better system design: we derive several insights that can help us to design a better cognitive radio system. For example, the efficiency loss can be mitigated if we can control the number of SUs competing for access or the heterogeneity of expected rates for different channels.

The outline of this paper is as follows. The system model for the multiuser multichannel cognitive MAC game is described in Section II. In Section III, we show that our game is in fact a congestion game with several nice properties. The analysis and computation of PoA for both symmetric and asymmetric games are given in Sections IV and V, respectively. Conclusion and extension work are discussed in Section VI. 
Slot 1

$\mathrm{PU}$

Transmits

\begin{tabular}{|l|l|l|l|l|}
\hline SUS & Silence & SS & CR & One SU Transmits \\
\hline
\end{tabular}

Fig. 1. Activities of PU and SUs of a channel in different time slots. In Slot 1, SUs can only keep silence after sensing that PU is transmitting; while in Slot 2, SUs sense that PU does not transmit. All users would undergo the contention resolution process and only one of the SUs is allowed to transmit in the remaining time of the slot.

\section{Cognitive MAC Game Model}

Consider a cognitive radio network model where there are a total of $M$ channels (owned by $M$ PUs) and $N$ SUs. The expected data rate of channel $j$ perceived by $\mathrm{SU} i$ is $R_{i j} .{ }^{1} \mathrm{We}$ assume $R_{i j}$ s for all SUs and channels are common knowledge of all SUs. The analysis of incomplete information game is part of our ongoing work.

The time is divided into discrete slots. The PU of a particular channel may transmit or be silent in any given time slot. The decision is made according to the PU's own communication objective without considering the activities of the SUs. We assume that all SUs can hear each other, and only one SU is allowed to transmit on a particular time in any time slot (if the corresponding PU is silent). For each channel, a time slot is divided into three stages: Sensing(SS), Contention Resolution(CR), and Transmission.

- Sensing (SS): At the beginning of a slot, SUs are refrained from accessing the channel and can only passively sense the channel for the presence of a PU. This can ensure the strict priority of the PU in terms of accessing the channel licensed to him.

- Contention Resolution (CR): Once the sensing period is over and no PU's activity is detected, a SU who has sensed this channel will pick a random starting time (i.e., a countdown value within a fixed time window) and continue to sense the channel for presence of other SUs.

- Transmission: A SU will proceed to transmit if its countdown timer expires and no other SUs transmit on the channel. Otherwise, if the channel is being used, the SU loses the opportunity to sense or transmit in other channels and remains idle till the next slot.

Due to the limitation of the hardware capability, we assume that each SU can only sense one channel in a time slot and transmit on the same channel if it is idle. A SU's goal is to choose a channel to sense in order to maximize his expected data rate. Such optimization not only depends on $R_{i j} \mathrm{~s}$ but also on the number of users competing for the same channel. Since each channel is available to only one SU after the CR phase, each SU sensing channel $j$ has a probability $1 / n_{j}$ to succeed in his transmission, where $n_{j}$ is the number of SUs sensing channel $j$. This motivates us to model and analyze

\footnotetext{
${ }^{1}$ Different SUs may achieve different data rates by using the same spectrum resource due to different choices of technologies.
}

TABLE I

KEY NOTATIONS IN THIS PAPER

\begin{tabular}{c|c}
\hline Notation & Physical Meaning \\
\hline \hline$M$ & the number of channels in the system \\
\hline $\mathcal{M}$ & the set of common channels \\
\hline $\mathcal{N}$ & the number of secondary users in the system \\
\hline$\Sigma_{i}$ & the set of secondary users \\
\hline$\sigma_{i}$ & strategy played by user $i$ \\
\hline $\boldsymbol{\sigma}$ & strategy profile of all users \\
\hline$\Pi$ & the number of users sensing channel $j$ \\
\hline$n_{j}$ & the expected rate of channel $j$ perceived by user $i$ \\
\hline$R_{i j}$ & payoff function of user $i$ for choosing channel $j$ \\
\hline$\pi_{i j}$ & total rates received by all users at a Nash Equilibrium $\boldsymbol{\sigma}$ \\
\hline$S U M(\boldsymbol{\sigma})$ & social optimum: maximum total rates received by all users \\
\hline$o p t$ & an indicator if channel $j$ is sensed \\
\hline$I_{j}$ & the set of $h$ channels not sensed by any user \\
\hline $\mathcal{H}$ & rate of the high-rate channels in a two-rate symmetric game \\
\hline$R$ & an indicator for channel heterogeneity \\
\hline$\alpha$ &
\end{tabular}

the cognitive MAC game as a congestion game as described in Section III

\section{Congestion Game Formulation}

We now model our cognitive random access system as a non-cooperative game. The key notations of this paper are listed in Table I. Consider the game tuple $\left(\mathcal{N}, \mathcal{M},\left(\Sigma_{i}\right)_{i \in \mathcal{N}},\left(\pi_{i j}\right)_{i \in \mathcal{N}, j \in \mathcal{M}}\right)$, where $\mathcal{N}=\{1, \ldots, N\}$ is the set of SUs, $\mathcal{M}=\{1, \ldots, M\}$ is the set of channels, and $\Sigma_{i}$ is the set of pure strategies for user $i$. Since SUs have the same set of channels to sense, we have $\Sigma_{i}=\mathcal{M}$ for all $i$. Finally, $\pi_{i j}=R_{i j} / n_{j}$ is the payoff function of SU $i$ for sensing channel $j$, which is monotonically decreasing in the number of users on channel $j, n_{j}$. Each SU $i$ wants to choose a channel to maximize his expected data rate, i.e., $\max _{j \in \Sigma_{i}} \pi_{i j}$. Since primary users do not appear explicitly in the description and analysis of the game, from now on we will simply use "user" to denote "secondary user".

A pure strategy profile is given by $\boldsymbol{\sigma}=\left(\sigma_{1}, \ldots, \sigma_{N}\right)$, where $\sigma_{i} \in \Sigma_{i}$ denotes the channel that user $i$ senses. The set of strategy profiles is denoted by $\Pi=\Sigma_{1} \times \Sigma_{2} \times \ldots \times \Sigma_{N}$. The profile $\boldsymbol{\sigma}$ is a Nash Equilibrium if and only if no user can improve his payoff by deviating unilaterally, i.e.,

$$
\frac{R_{i \sigma_{i}}}{n_{\sigma_{i}}} \geq \frac{R_{i k}}{n_{k}+1}, \quad \forall i \in \mathcal{N}, \forall k \neq \sigma_{i} .
$$

\section{A. Existence and Uniqueness of the Nash Equilibrium}

Our cognitive MAC game actually belongs to the class of congestion games [7], [8], which always has a pure strategy Nash Equilibrium. This can be proved by showing that there exists an exact potential function of the game, and such function is upper-bounded and keeps increasing when users update their strategies greedily and myopically, and thus eventually a Nash Equilibrium must be reached.

On the other hand, it is quite common to have multiple Nash Equilibria in a congestion game, which is the case for 
TABLE II

PRICE OF ANARCHY FOR VARIOUS COGNITIVE MAC GAMES IN THIS PAPER

\begin{tabular}{c|ccc|cc}
\hline & \multicolumn{3}{|c|}{ Symmetric MAC game (Section IV) } & Asymmetric MAC game (Section V) \\
\hline \hline & Identical & Two-rate & General & Bounded \\
\hline \hline Parameters & $M, N$ & $M, N, R$ & $M, N$ & $M, N, \alpha$ & $M, N$ \\
\hline$P o A$ & 1 & equations (1) and (2) & $\frac{N}{N+\min (N, M)-1}$ & $\frac{\alpha+N-1}{\alpha N+\min (N, M)-1}$ \\
\hline $\min _{M, N}$ PoA & 1 & $\frac{R}{2 R-1}$ & $\frac{1}{2}$ & $\frac{1}{\alpha+1}$ & $\frac{1}{N}$ \\
\hline
\end{tabular}

our game as well ${ }^{2}$. In this paper, we focus our analysis on the pure strategy Nash Equilibria ${ }^{3}$.

\section{B. Efficiency of the Nash Equilibrium}

Given at least one Nash Equilibrium exists in our game, a natural question to ask is how well this Nash Equilibrium performs. The way to quantify this is the Price of Anarchy (PoA) defined next.

Let us first represent the total expected rate received by all users at a given Nash Equilibrium $\boldsymbol{\sigma}$ as

$$
S U M(\boldsymbol{\sigma})=\sum_{i \in \mathcal{N}} \pi_{i \sigma_{i}} .
$$

The social optimum opt of the game is defined to be the maximum total rates received by the $N$ users ${ }^{4}$.

Definition 1 (Efficiency Ratio): The efficiency ratio of a Nash Equilibrium $\sigma$ is the ratio between the total expected rate received by all users at $\sigma$ and the social optimum,

$$
E R(\boldsymbol{\sigma})=\frac{S U M(\boldsymbol{\sigma})}{o p t} .
$$

Definition 2 (PoA): The price of anarchy of a family of games $^{5}$ is the worst-case efficiency ratio among all pure strategy Nash Equilibria,

$$
P o A=\min _{\boldsymbol{\sigma} \in \Pi} \frac{S U M(\boldsymbol{\sigma})}{o p t}=\min _{\boldsymbol{\sigma} \in \Pi} E R(\boldsymbol{\sigma}) .
$$

To facilitate the study of PoA, we classify the cognitive MAC game into several families of games depending on the heterogeneity of users and channels.

- Symmetric game: all users have the same expected rate for any given channel, i.e., $R_{i j}=R_{j}$ for all user $i \in \mathcal{N}$ and all channel $j \in \mathcal{M}$.

- Identical game: a special case of symmetric game where all channels are the same, i.e., $R_{i j}$ is a constant for all user $i \in \mathcal{N}$ and all channel $j \in \mathcal{M}$.

- Two-rate symmetric game: another special case of symmetric game where the rates of channels take only two values (high or low).

\footnotetext{
${ }^{2}$ Imagine two identical users sensing two identical channels, and there will be two pure strategy Nash Equilibria.

${ }^{3}$ Mixed strategies are more difficult to analyze and less attractive in terms of practical implementations.

${ }^{4}$ Such social optimum could be achieved, for example, through a coordinated random access protocols. We notice that each user still chooses one channel to sense, thus congestion can not be completely avoided even at a socially optimal solution.

${ }^{5} \mathrm{We}$ will discuss different families of games in this paper and the specific meaning will be clear later.
}

- Asymmetric game: the most general case where $R_{i j}$ can be different for each $i$ and $j$.

- Bounded Asymmetric game: a special case of asymmetric game where the heterogeneity between channels is bounded by a finite value $\alpha=\max _{j}\left(\max _{i, k} \frac{R_{i j}}{R_{k j}}\right)$.

The key results of the PoA for different games are summarized in Table II.

\section{PoA Analysis of Symmetric Games}

\section{A. Identical and General Symmetric Games}

In symmetric games, each channel $j$ has the same expected rate $R_{j}$ for all users, but different channels can have different rates. Let us first look at the properties of a Nash Equilibrium and a socially optimal solution in this case.

For each channel $j$, we use an indicator $I_{j}$ to represent whether this channel is sensed by some users,

$$
I_{j}= \begin{cases}1, & \text { if } n_{j}>0 \\ 0, & \text { if } n_{j}=0 .\end{cases}
$$

Thus the total rate achieved at a Nash Equilibrium $\boldsymbol{\sigma}$ in a symmetric game is

$$
S U M(\boldsymbol{\sigma})=\sum_{j \in \mathcal{M}} R_{j} I_{j} .
$$

The social optimum in a symmetric game can be calculated as follows. If the number of users is no less than the number of channels $(N \geq M)$, the social optimum is reached when all channels are being sensed by some SUs and opt $=\sum_{j \in \mathcal{M}} R_{j}$. While $N<M$, each user should sense a different channel at a socially optimal solution. Such channels should be those with the largest $N$ channels in terms of expected rates.

To facilitate the discussion, we use $(N, M)$-game to represent a game with $N$ users and $M$ channels. First consider the identical game, which is a special case of the symmetric game.

Theorem 1: For an identical $(N, M)$-game, the PoA always equals 1.

Proof: Since all channels are identical with rate $R$, each user will sense the least congested channel at a Nash Equilibrium. For $N \geq M$, all $M$ channels will be sensed; while for $N<M, N$ channels will be sensed with one user per channel. The total rates obtained at a Nash Equilibrium is the same as that at the social optimum in both cases.

Theorem 2: For a symmetric $(N, M)$-game with more users than number of channels $(N \geq M)$, the PoA is $\frac{N}{N+M-1}$.

Proof: The proof is divided into two steps. 
Step I (lower bound): we first prove that the efficiency ratio of a Nash Equilibrium in this case is lower bounded by $\frac{N}{N+M-1}$. To begin with, we assume that there exists a Nash Equilibrium $\boldsymbol{\sigma}$ with $h(<M)$ channels not sensed by any user. Without loss of generality, assume that the channels are indexed such that $R_{1} \geq R_{2} \geq \cdots \geq R_{M}$. Thus, we can have the following:

1) The last $h$ channels are not sensed by any users. Define set $\mathcal{H}=\{M-h+1, \ldots, M\}$. Then, we have $I_{j}=0$ for $j \in \mathcal{H}$.

2) Each of the $N$ users senses one of the first $M-h$ channels, i.e., $\sum_{j \in \mathcal{M} \backslash \mathcal{H}} n_{j}=N$.

3) Each user chooses his best-reply strategy at this Nash Equilibrium, i.e., $\frac{R_{j}}{n_{j}} \geq R_{k}$ for all $j \in \mathcal{M} \backslash \mathcal{H}$ and $k \in \mathcal{H}$. This implies $\frac{R_{j}}{n_{j}} \geq \frac{1}{h} \sum_{k \in \mathcal{H}} R_{k}$ for all $j \in \mathcal{M} \backslash \mathcal{H}$.

Now we can derive the lower bound of the efficiency ratio

$$
\begin{aligned}
E R(\boldsymbol{\sigma}) & =\frac{S U M(\boldsymbol{\sigma})}{o p t}=\frac{\sum_{j \in \mathcal{M}} R_{j} I_{j}}{\sum_{j \in \mathcal{M}} R_{j}}=\frac{\sum_{j \in \mathcal{M} \backslash \mathcal{H}} R_{j}}{\sum_{j \in \mathcal{M}} R_{j}} \\
& =1-\frac{\sum_{j \in \mathcal{H}} R_{j}}{\sum_{j \in \mathcal{M} \backslash \mathcal{H}} R_{j}+\sum_{j \in \mathcal{H}} R_{j}} \\
& \geq 1-\frac{\sum_{j \in \mathcal{H}} R_{j}}{\sum_{j \in \mathcal{M} \backslash \mathcal{H}}\left(\frac{n_{j}}{h} \sum_{k \in \mathcal{H}} R_{k}\right)+\sum_{j \in \mathcal{H}} R_{j}} \\
& =1-\frac{\sum_{j \in \mathcal{H}} R_{j}}{\frac{1}{h} \sum_{k \in \mathcal{H}} R_{k}\left(\sum_{j \in \mathcal{M} \backslash \mathcal{H}} n_{j}\right)+\sum_{j \in \mathcal{H}} R_{j}} \\
& =1-\frac{\sum_{j \in \mathcal{H}} R_{j}}{\frac{N}{h} \sum_{k \in \mathcal{H}} R_{k}+\sum_{j \in \mathcal{H}} R_{j}} \\
& =1-\frac{1}{\frac{N}{h}+1}=\frac{N}{N+h} .
\end{aligned}
$$

The above analysis is for a fixed $h$. It is clear that the lower bound $\frac{N}{N+h}$ is a decreasing function of $h$, and achieves its minimum value of $\frac{N}{N+M-1}$ when there are $h=M-1$ channels not sensed at a Nash Equilibrium $\sigma$.

Step II (achievability): we construct a symmetric $(N, M)$ game that achieves the efficiency ratio $\frac{N}{N+M-1}$. Consider a game with $M-1$ identical channels having the same rate $R_{j}=R$. The remaining one channel has a rate of $N R$. A Nash Equilibrium $\sigma$ of this game is that all users sense the channel with largest expected rate. The efficient ratio of $\sigma$ is

$$
E R(\boldsymbol{\sigma})=\frac{S U M(\boldsymbol{\sigma})}{o p t}=\frac{N R}{N R+(M-1) R}=\frac{N}{N+M-1} .
$$

Combining both steps and the Definition 2 of PoA, we have proved the theorem.

Theorem 3: For a symmetric $(N, M)$-game with less users than number of channels $(N<M)$, the PoA is $\frac{N}{2 N-1}$.

Proof: First arrange the channels in a descending order of rates. Since the last $M-N$ channels will not be used either in the socially optimal solutions or any Nash Equilibrium, we can safely discard them. Therefore, the problem is reduced to a symmetric $(N, N)$-game and Theorem 2 applies.

Remark 1: (Asymptotic PoA) With Theorems 2 and 3, we know that the PoA for any symmetric $(N, M)$-game is lower bounded by $\frac{1}{2}$ when $N<M$. When $N \geq M$, it is possible to achieve PoA=1 when $N$ goes to infinity while $M$ keeps as a constant. In this case, all channels are sensed by some users. This is because we only allow one user to transmit over a channel, and thus there is no loss in terms of total expected achievable rate if more than one user sense the same channel (i.e., $n_{j} \frac{R_{j}}{n_{j}}=R_{j}$ ).

Remark 2: (Insight of System Design) Step II of Theorem 2's proof suggests that the worst case efficiency loss happens when all users choose to sense one single "big" channel at the Nash Equilibrium. This motivates us to make sure that channels are of similar rates in a cognitive system if possible. In particular, it is better to group small channels together so that they will not be left unused. The intuition is that each user can sense one channel only and channels with very small rates will never be chosen.

\section{B. Two-Rate Symmetric Games}

The PoA results in Theorems 2 and 3 might be too pessimistic in practice since the rates of different channels maybe more consistent in practice. In particular, there seldom exists one channel that outperforms all other channel with a extremely high transmission rate.

To gain more insights into the PoA for the less extreme cases, we study a simple symmetric game model where there exists two types of channels: $L$ channels have equal normalized rates of $R_{j}=1$, and the remaining $M-L$ channels have the same high rate of $R_{j}=R>1$. For simplicity, $R$ is taken to be an integer. We call such a game two-rate symmetric game with parameters $(M, N, L, R)$. The goal of this analysis is to study how PoA varies with the parameters in this case, in particular, the heterogeneity parameter $R$ between different channels.

Theorem 4: Consider a two-rate symmetric game with parameters $(M, N, L, R)$. If $(M-L) R \leq M$,

$\operatorname{Po} A= \begin{cases}1, & \text { if } N \leq M-L \\ \frac{(M-L) R}{(M-L) R+(N-M+L)}, & \text { if } M-L<N \leq(M-L) R \\ \frac{N}{(M-L) R+(N-M+L)}, & \text { if }(M-L) R<N \leq M \\ \frac{N}{(M-L) R+L}, & \text { if } M<N \leq(M-L) R+L \\ 1, & \text { if } N>(M-L) R+L\end{cases}$

If $(M-L) R>M$,

$\operatorname{PoA}= \begin{cases}1, & \text { if } N \leq M-L \\ \frac{(M-L) R}{(M-L) R+(N-M+L)}, & \text { if } M-L<N \leq M \\ \frac{(M-L) R}{(M-L) R+L}, & \text { if } M<N \leq(M-L) R \\ \frac{N}{(M-L) R+L}, & \text { if }(M-L) R<N \leq(M-L) R+L \\ 1, & \text { if } N>(M-L) R+L\end{cases}$

Proof: When $M, L$ and $R$ are given, the total rates achieved at the social optimum and the unique Nash Equilibrium can be explicitly calculated as summarized in Figures 2 and 3 for different $N$. The values under the axis are threshold values of $N$, and the values above the axis are total rates. Since the PoA is the worst case ratio of total rate at a Nash 


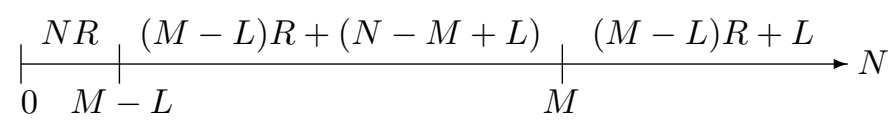

Fig. 2. Total rate at the social optimum for different $\mathrm{N}$

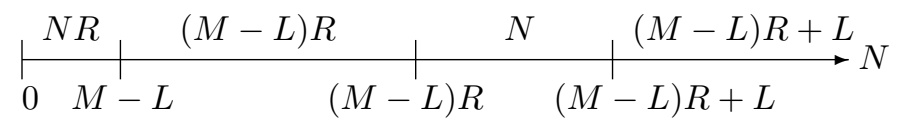

Fig. 3. Worst total rate at NE for different $\mathrm{N}$

Equilibrium over the social optimum, we can get the closedform solution for PoA by combining the two figures.

Theorem 5: In a two-rate symmetric game with parameters $(M, N, L, R)$, the worst possible PoA is $\frac{R}{2 R-1}$ for any possible $M, N$ and $L$.

Proof: The minimum PoA can be derived from the results in Theorem 4. For $(M-L) R \leq M$, minimum PoA of $\frac{R}{2 R-1}$ is obtained at $N=(M-L) R$; while for $(M-L) R>M$, minimum PoA of $\frac{(M-L) R}{(M-L) R+L}$ is obtained for $M<N \leq(M-$ $L) R$. It can be shown that minimum PoA of $\frac{(M-L) R}{(M-L) R+L}$ is greater than $\frac{R}{2 R-1}$ for $(M-L) R>M$. Therefore, the worst possible PoA of this game is $\frac{R}{2 R-1}$.

\section{PoA Analysis of Asymmetric Games}

\section{A. General Asymmetric Games}

In an asymmetric game, different users can have different evaluations on the same channel, e.g., due to different technologies used on that spectrum. We first study how channels are sensed by users at a socially optimal solution before computing the PoA.

Lemma 1: For an asymmetric $(N, M)$-game, $\min (N, M)$ of channels are sensed at a socially optimal solution.

Proof: [Sketch] First, we prove that for an asymmetric game with $M=2$ and $N \geq 2$, the social optimum is obtained if and only if both channels are sensed. This can be proved by contradiction, i.e., by showing that it is always possible to increase the total rate of all users when a user deviates to sense a channel with no SUs if such channel exists.

Then we can use this fact to show the following: for $N \geq$ $M$, all $M$ channels are sensed at any socially optimal solution; while for $N<M$, exactly $N$ channels are sensed at any socially optimal solution. The whole proof is given in [20].

Lemma 1 characterizes the properties of all socially optimal solutions without precisely describing the user-channel associations. Such partial characterization, however, turns out to be sufficient for the later analysis of PoA.

We are ready to analyze the PoA now. For the case of single channel $(M=1)$, all users have no choice but sense the same channel only. Therefore, the total payoff obtained at a Nash Equilibrium is the same as the social optimum and the PoA is 1 . We then consider the more interesting cases with more than one channel.

Theorem 6: For an asymmetric $(N, M)$-game with $M>1$, the PoA is $\frac{1}{N}$.

Proof: [Sketch] We consider two cases separately: $N \leq$ $M$ and $N>M$. The key idea is similar as the proof of
Theorem 2. We first show that the efficiency ratio of a Nash Equilibrium in any asymmetric game is lower-bounded by $\frac{1}{N}$. Then we show that for any $\delta>0$, a game with efficiency ratio $\frac{1}{N}+\delta$ can be constructed. Taking the limit of $\delta$ to be zero and we obtain the PoA of $\frac{1}{N}$. A brief outline of the proof for the case $N>M$ is given in the Appendix and the complete proof is given in [20].

Remark 3: (Asymptotic PoA) It is interesting to notice that the PoA in the asymmetric case is $\frac{1}{N}$ and thus independent of the number of channels $M$. Also, for very large $N$, the PoA can be arbitrarily small. This result is different from that in symmetric games, in which a large $N$ actually improves the performance of a Nash Equilibrium (in terms of PoA).

Remark 4: (Insight of System Design) In order to lower bound the PoA, we may want to limit the number of users competing in a network. In the scenario where we have a large population of SUs competing for the access of channels, we may want to segment them into several different systems (i.e., each user is only allowed to choose from a subset of channels instead of all channels). Moreover, channels of similar rates should be put in the same system. This can help to prevent the case where all users sense the same very large channel (as shown to be the worst case in the proof).

\section{B. Bounded Asymmetric Games}

The PoA of $\frac{1}{N}$ obtained in a general asymmetric games may approach 0 for sufficiently large number of users $N$. However, this worst case happens rarely in practice. This motivates our ongoing work of finding the PoA when the largest discrepancy of expected rates between users across all channels is bounded. Mathematically, we denote such discrepancy as

$$
\alpha=\max _{j}\left(\max _{i, k} \frac{R_{i j}}{R_{k j}}\right) .
$$

Our preliminary results show that PoA could be written as a function of $N, M$ and $\alpha$ for an asymmetric game bounded with parameter $\alpha$ (as shown in Table II), which is lowerbounded by $\frac{1}{\alpha+1}$ for any $N$ and $M$. This result provides a finer granularity description about the PoA of asymmetric games. This also coincides with the $1 / 2$ result described in Remark 1 for the general symmetric case, which is a special case of the asymmetric game with $\alpha=1$.

\section{CONClusion ANd Future Work}

In this paper, we study the performance of a multi-user multi-channel cognitive MAC game. After showing that a Nash Equilibrium exists for the game, we focus on the study of the worst case efficiency ratio of a Nash Equilibrium comparing with the optimal performance achieved under a coordinated random MAC protocol, i.e., the price of anarchy.

The calculation of PoA is non-trivial since characterizing either the Nash Equilibrium or the social optimum is difficult in the general case. Despite the difficulties, we are able to give closed-form PoA expressions for various symmetric and asymmetric games with arbitrary number of users and channels. The key results are summarized in Table II. 
Moreover, we are able to identify the scenarios under which the worst possible Nash Equilibrium happens. This leads to useful insights on how we should design the cognitive radio systems such that the efficiency loss is not too severe. In general, we should avoid having channels with very different expected data rates in the same system and should avoid too many users accessing a limited number of channels.

Several interesting directions remain widely open. For example, how large is PoA when we consider mixed strategy Nash Equilibrium? How to analyze a system where a user can sense more than one channel in each time slot? How the results change when different users can only sense different subsets of the channels? Finally, what if we allow multiple SUs to transmit over one channel simultaneously?

\section{APPENDIX}

\section{A. Proof outline of Theorem 6 for the case of $N>M$}

First we give the lower-bound of the efficiency ratio. For an equilibrium $\boldsymbol{\sigma}=\left(\sigma_{1}, \sigma_{2}, \ldots, \sigma_{N}\right)$, we have $\frac{R_{i \sigma_{i}}}{n_{\sigma_{i}}} \geq \frac{R_{i j}}{n_{j}+1}$ for all $i \in \mathcal{N}$ and $j \neq \sigma_{i}$. Here $\boldsymbol{n}=\left(n_{1}, n_{2}, \ldots, n_{M}\right)$ is the congestion vector of $\sigma$, which is a vector that shows the number of users sensing on each channel. We will also denote $\boldsymbol{\omega}=\left(\omega_{1}, \omega_{2}, \ldots, \omega_{N}\right)$ as the strategy profile played by the users at a socially optimal solution, and $\boldsymbol{m}=\left(m_{1}, m_{2}, \ldots, m_{M}\right)$ be its corresponding congestion vector .

By using the inequality $\frac{\sum_{i} a_{i}}{\sum_{i} b_{i}} \geq \min _{i} \frac{a_{i}}{b_{i}}$, we have

$$
\operatorname{ER}(\sigma)=\frac{\sum_{i=1}^{N} R_{i \sigma_{i}} / n_{\sigma_{i}}}{\sum_{i=1}^{N} R_{i \omega_{i}} / m_{\omega_{i}}} \geq \min _{i} \frac{R_{i \sigma_{i}} / n_{\sigma_{i}}}{R_{i \omega_{i}} / m_{\omega_{i}}} .
$$

Assume $\bar{i}=\arg \min _{i} \frac{R_{i \sigma_{i}} / n_{\sigma_{i}}}{R_{i \omega_{i}} / m_{\omega_{i}}}$, we have two possible cases:

- If $\sigma_{\bar{i}}=\omega_{\bar{i}}$, then $\min _{i} \frac{R_{i \sigma_{i}} / n_{\sigma_{i}}}{R_{i \omega_{i}} / m_{\omega_{i}}}=\frac{m_{\omega_{\bar{i}}}}{n_{\omega_{\bar{i}}}} \geq \frac{1}{N}$.

- If $\sigma_{\bar{i}} \neq \omega_{\bar{i}}$, then $\min _{i} \frac{R_{i \sigma_{i}} / n_{\sigma_{i}}}{R_{i \omega_{i}} / m_{\omega_{i}}} \geq \frac{m_{\omega_{\bar{i}}}}{n_{\omega_{\bar{i}}}+1} \geq \frac{1}{N}$. The first inequality is due to the best-reply strategy of user $\bar{i}$.

In both cases, the efficiency ratio is lower-bounded by $\frac{1}{N}$.

We now construct an asymmetric $(N, M)$-game with efficiency ratio $\frac{1}{N}+\delta$ for any $\delta>0$. Different values of $R_{i j}$ of such a game are shown in Table III. The $i$ th row represents the rates of all channels perceived by user $i$, and column $j$ represents the rates of all users on channel $j$. With the condition $\frac{R}{N}>\epsilon$, the social optimum is achieved when the first user senses channel 1 and all the remaining $M-1$ channels are sensed by the remaining $N-1$ users. We can also identify one Nash Equilibrium $\sigma$ where all users sense channel 1 . Therefore, we have

$$
E R(\boldsymbol{\sigma})=\frac{\frac{R+(N-1) \epsilon}{N}}{R+\frac{(M-1)}{N+1} \epsilon}=\frac{1}{N}+\frac{\frac{N^{2}-M}{N(N+1)} \epsilon}{R+\frac{(M-1)}{N+1} \epsilon}=\frac{1}{N}+\delta,
$$

where $\delta=\frac{\frac{N^{2}-M}{N(N+1)} \epsilon}{R+\frac{(M-1)}{N+1} \epsilon}$. When $\epsilon$ goes to zero, $\delta$ goes to zero and the PoA equals $\frac{1}{N}$.
TABLE III

EXample of An Asymmetric Game with EFficiency Ratio $\frac{1}{N}+\delta$

\begin{tabular}{c|cccc} 
& 1 & 2 & $\cdots$ & $M$ \\
\hline 1 & $R$ & $\frac{R}{N}$ & $\cdots$ & $\frac{R}{N}$ \\
2 & $\epsilon$ & $\frac{\epsilon}{N+1}$ & $\cdots$ & $\frac{\epsilon}{N+1}$ \\
$\vdots$ & $\vdots$ & & & $\vdots$ \\
$N$ & $\epsilon$ & $\frac{\epsilon}{N+1}$ & $\cdots$ & $\frac{\epsilon}{N+1}$
\end{tabular}

\section{REFERENCES}

[1] R. Etkin, A. Parekh, and D. Tse, "Spectrum sharing for unlicensed bands," IEEE Journal on Selected Areas in Communications, vol. 25, no. 3, pp. 517 - 528, Apr 2007.

[2] R. Menon, R. Buehrer, and J. Reed, "Outage probability based comparison of underlay and overlay spectrum sharing techniques," IEEE International Symposium on New Frontiers in Dynamic Spectrum Access Networks (DySPAN), pp. 101 - 109, 2005.

[3] V. Chakravarthy, Z. Wu, A. Shaw, M. Temple, R. Kannan, and F. Garber, "A general overlay/underlay analytic expression representing cognitive radio waveform," Waveform Diversity and Design Conference, 2007. International, pp. 69 - 73, 2007.

[4] P. Papadimitratos, S. Sankaranarayanan, and A. Mishra, "A bandwidth sharing approach to improve licensed spectrum utilization," IEEE Communications Magazine, vol. 43, no. 12, pp. supl.10 - supl.14, Dec 2005.

[5] J. Zhao, H. Zheng, and G.-H. Yang, "Distributed coordination in dynamic spectrum allocation networks," IEEE International Symposium on New Frontiers in Dynamic Spectrum Access Networks (DySPAN), pp. $259-$ 268, Oct 2005.

[6] Q. Zhao, L. Tong, A. Swami, and Y. Chen, "Decentralized cognitive mac for opportunistic spectrum access in ad hoc networks: A POMDP framework," IEEE Journal on Selected Areas in Communications, vol. 25, no. 3, pp. 589 - 600, Apr 2007.

[7] R. Rosenthal, "A class of games possessing pure-strategy Nash equilibria," International Journal of Game Theory, vol. 2, pp. 65-67, 1973.

[8] I. Milchtaich, "Congestion Games with Player-Specific Payoff Functions," Games and Economic Behavior, vol. 13, no. 1, pp. 111-124, 1996.

[9] B. Vocking, "Congestion games: Optimization in competition," Proceedings of the 2nd Algorithms and Complexity in Durham Workshop, Jan 2006.

[10] G. Christodoulou and E. Koutsoupias, "The price of anarchy of finite congestion games," In Proceedings of the 37th Annual ACM Symposium on Theory and Computing (STOC-05), 2005.

[11] R. Johari and J.N. Tsitsiklis, "A scalable network resource allocation mechanism with bounded efficiency loss," IEEE Journal on Selected Areas in Communications, 24(5):992-999, 2006.

[12] M. Liu and Y. Wu, "Spectrum Sharing as Congestion Games," in Proc. 46th Allerton Conf. Comm. Control and Computing, Monticello, IL,Sept. 2008.

[13] I. Akyildiz, W. Lee, M. Vuran, and S. Mohanty, "Next generation/dynamic spectrum access/ cognitive radio wireless networks: A survey," Computer Networks, Jan 2006.

[14] Q. Zhao and B. Sadler, "A survey of dynamic spectrum access," IEEE Signal Processing Magazine, vol. 24, no. 3, pp. 79 - 89, May 2007.

[15] Y. Chen and J. Zhang, "Design of price mechanisms for network resource allocation via price of anarchy," Operations Research (to appear), 2009.

[16] T. Harks and K. Miller, "Efficiency and stability of nash equilibria in resource allocation games," Technical Report, Department of Mathematics, Technische Universitt Berlin, 2008.

[17] T. Roughgarden and E. Tardos, "How bad is selfish routing," Journal of the ACM, vol. 49, pp. 236-259, Feb. 2002.

[18] R. Johari, S. Mannor, and J. N. Tsitsiklis, "Efficiency loss in a network resource allocation game: the case of elastic supply," IEEE Trans. on Automatic Control, vol. 50, no. 11, pp. 1712-1724, Nov 2005.

[19] S. Huang, X. Liu, and Z. Ding, "Opportunistic spectrum access in cognitive radio networks," IEEE INFOCOM, 2008.

[20] L. Law, J. Huang, M. Liu, and S. Li, "Price of Anarchy for Cognitive MAC Games," technical report, http://www.ie.cuhk.edu.hk/ $\sim$ jwhuang/publication/PoA_TechReport.pdf. 